\title{
Long noncoding RNA MALAT1 inhibits apoptosis induced by oxygen-glucose deprivation and reoxygenation in human brain microvascular endothelial cells
}

\author{
JIA-WEI XIN and YU-GANG JIANG
}

Department of Neurosurgery, The Second Xiangya Hospital, Central South University, Changsha, Hunan 410011, P.R. China

Received March 9, 2015; Accepted July 7, 2016

DOI: 10.3892/etm.2017.4095

\begin{abstract}
Cerebral ischemia/reperfusion (I/R) injury leads to brain vascular dysfunction, which is characterized by endothelial cell injury or death. Long noncoding (lnc) RNA metastasis-associated lung adenocarcinoma transcript 1 (MALAT1) is reportedly associated with endothelial cell functions and dysfunctions. In the present study, the role of MALAT1 in I/R-induced cerebral vascular endothelial cell apoptosis was explored using oxygen-glucose deprivation and reoxygenation (OGD-R) as an in vitro I/R injury model. Primary human brain microvascular endothelial cells were cultured under OGD-R, and the expression levels of MALAT1 and cell apoptosis were measured at 6, 9, 12, 24 and $36 \mathrm{~h}$ post-reoxygenation. The expression levels of MALAT1 and the apoptotic rate of cells exposed to OGD-R exhibited contrasting trends following reoxygenation. Following OGD-R, lentiviral overexpression of MALAT1 increased phosphatidylinositol 3-kinase (PI3K) activities and the activation of Akt phosphorylation, and decreased cell apoptosis and caspase 3 activities, which were successfully abolished by treatment with a PI3K inhibitor, Wortmannin. Conversely, lentiviral knockdown of MALAT1 decreased PI3K activities and the activation of Akt phosphorylation, and increased cell apoptosis and caspase 3 activity. Overexpression and knockdown of MALAT1 exhibited no significant effects on OGD-R-induced reactive oxygen species (ROS) production. In conclusion, to the best of our knowledge, the present study was the first to suggest that lncRNA MALAT1 may protect human brain vascular endothelial cells from OGD-R-induced apoptosis via a PI3K-dependent mechanism. These findings
\end{abstract}

Correspondence to: Professor Yu-Gang Jiang, Department of Neurosurgery, The Second Xiangya Hospital, Central South University, 139 Middle Renmin Road, Changsha, Hunan 410011, P.R. China

E-mail: 13707315567@139.com

Key words: long noncoding RNA, metastasis-associated lung adenocarcinoma transcript 1, apoptosis, ischemia/reperfusion, oxygen-glucose deprivation and deoxygenation, endothelial cell, phosphatidylinositol 3-kinase, Akt suggest that MALAT1 may be a potential novel therapeutic target for cerebral I/R injury.

\section{Introduction}

Ischemia/reperfusion (I/R) injury has previously been demonstrated to be the cause of debilitating complications and mortality associated with stroke, myocardial infarction and traumatic brain injury (1). I/R injury in cerebral vessels is characterized as an early secondary injury leading to inflammation and edema (1). Cerebral hypoxia and ischemia can lead to vascular cell damage, which manifests as impairments in autoregulation and vascular reactivity and endothelial cell apoptosis (2). Morbidity from reperfusion and post-ischemia damage remains common, despite improvements in imaging, interventional techniques and pharmacological agents. Cerebral I/R injury activates various distinct, yet overlapping, cell signaling pathways, which lead to vascular dysfunction characterized by endothelial cell injury or apoptosis (2).

A large proportion $(>80 \%)$ of the human genome is transcribed; however, only $<2 \%$ is subsequently translated into proteins, suggesting that the vast majority of DNA sequences are transcribed as non-protein coding RNAs (3). A novel class of noncoding RNAs, which are $>200$ nucleotides and termed long noncoding RNAs (lncRNAs), have been characterized $(4,5)$. IncRNAs are localized in the nucleus or cytoplasm and are involved in the activation and inhibition of gene expression through epigenetic mechanisms, including chromatin remodeling, regulation of splicing, and by acting as sponges for microRNAs $(6,7)$. A recent study showed significant changes in the expression profiles of lncRNA after oxygen-glucose deprivation (OGD), suggesting a potential pathological role of lncRNAs in mediating endothelial responses to ischemic stimuli (8).

Metastasis-associated lung adenocarcinoma transcript 1 (MALAT1), which is a $6.5-\mathrm{Kb}$ nuclear residing lncRNA, was initially demonstrated to control tumor metastasis and cancer cell survival (9). Recent studies have shown that MALAT1 is enriched in endothelial cells and is closely associated with endothelial cell functions and dysfunctions, including hyperglycaemia-induced inflammatory processes (9), proliferation (10) and angiogenesis (11).

In the present study, the role of MALAT1 in I/R-induced cerebral vascular endothelial cell apoptosis was investigated, 
using OGD and reoxygenation (OGD-R) as an in vitro $\mathrm{I} / \mathrm{R}$ injury model (1).

\section{Materials and methods}

Establishment of an in vitro model of $O G D-R$. Primary human brain microvascular endothelial cells were purchased from Cell Systems Corp., (cat. no. ACBRI 376; Kirkland, WA, USA) and cultured in CSC Complete Medium, which includes $10 \%$ fetal bovine serum (cat. no. 4Z0-500; Cell Systems Corp.), in a humidified atmosphere (relative humidity $>95 \%$ ) containing $5 \% \mathrm{CO}_{2}$ at $37^{\circ} \mathrm{C}$. To avoid undue depletion of glucose in the culture medium, experiments were performed $24 \mathrm{~h}$ after the final medium renewal. To simulate ischemia-like conditions in vitro, cells were subjected to OGD as previously described (12). Cells were exposed to OGD for $4 \mathrm{~h}$ by replacing the culture medium with a glucose- and serum-free medium that had been equilibrated in an anaerobic atmosphere $(<0.1 \%$ $\mathrm{O}_{2}, 5 \% \mathrm{CO}_{2}$ and $95 \% \mathrm{~N}_{2}$ ) at $37^{\circ} \mathrm{C}$ inside a hypoxic workstation (H35; Don Whitley Scientific Ltd., Shipley, UK). The pH of the medium remained constant throughout the experiments. As reoxygenation consisted of a return to the initial culture conditions after OGD, cells were withdrawn from the hypoxic workstation and the OGD medium was replaced with glucoseand serum-containing culture medium. Cells grown under normal culture conditions were used as a control.

Lentiviral transduction. Human MALAT1 lentiviral vector (cat. no. LV212703) and blank control lentivector (cat. no. LV587) were purchased from Applied Biological Materials Inc., (Richmond, BC, Canada). MALAT1 and blank control lentivectors were transfected with the packaging vectors (psPAX2 and pMD2.G) into 293T cells by calcium chloride to produce the lentivirus. Primary human brain microvascular endothelial cells were subsequently transduced with the blank control or MALAT1-expressing lentivirus. Human MALAT1 siRNA lentivirus (cat. no. iV012699) and scrambled siRNA lentivirus (cat. no. LVP015-G) were purchased from Applied Biological Materials Inc., and were directly used to transduce primary human brain microvascular endothelial cells. Six hours after lentiviral transduction, cells were subjected to OGD-R.

Reverse transcription-quantitative polymerase chain reaction (RT-qPCR). Total RNA from cultured cells was isolated using miRNeasy kits (Qiagen China Co., Ltd., Shanghai, China) according to the manufacturer's protocol, followed by purification with TURBO DNA-free System (Ambion; Thermo Fisher Scientific, Inc., Waltham, MA, USA). To measure lncRNAs, 1,000 ng total RNA was reverse transcribed using MulV reverse transcriptase and random hexamer primers (both Thermo Fisher Scientific, Inc.) in a $20-\mu 1$ reaction. cDNA was used as a template for RT-qPCR using Fast SYBR Green and an Applied Biosystems StepOnePlus machine (both Applied Biosystems; Thermo Fisher scientific, Inc.). Human ribosomal P0 (RPLP0) mRNA was sequenced and used as a reference gene for normalization. Primer sequences were as follows: MALAT1, forward 5'-GTGATGCGAGTTGTTCTC CG-3' and reverse 5'-CTGGCTGCCTCAATGCCTAC-3'; and RPLP0, forward 5'-TCGACAATGGCAGCATCTAC-3' and reverse 5'-ATCCGTCTCCACAGACAAGG-3'. The PCR reaction mixture contined $12.5 \mu \mathrm{l}$ of SYBR Green Master Mix, $400 \mathrm{ng}$ of template DNA, forward and reverse primers $(0.25 \mu \mathrm{M}$ each), and $12 \mu \mathrm{l}$ of nuclease-free water. PCR amplification conditions were as follows: $20 \mathrm{sec}$ at $94^{\circ} \mathrm{C}$, followed by 40 cycles of $3 \mathrm{sec}$ at $95^{\circ} \mathrm{C}$ and $30 \mathrm{sec}$ at $62^{\circ} \mathrm{C}$, and a final extension at $62^{\circ} \mathrm{C}$ for 10 mins. Analysis of relative gene expression levels was performed using the following formula: $2^{-\Delta \Delta \mathrm{Cq}}$ with $\Delta \Delta \mathrm{CT}=\mathrm{Cq}_{\text {(target gene) }}-\mathrm{Cq}_{\text {(control) }}$ (13).

Cell apoptosis assay. Primary human brain microvascular

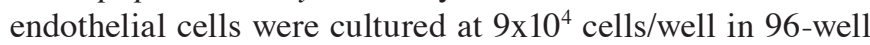
tissue culture plates and subjected to OGD-R. Cell apoptosis was measured at $6,9,12,24$ and $36 \mathrm{~h}$ post-reoxygenation with a microplate reader-based TiterTACS in situ apoptosis detection kit (cat. no. 4822-96-K; R\&D Systems, Inc., Minneapolis, MN, USA) according to the manufacturer's protocol. Each experiment was performed in duplicate and repeated thrice.

Terminal deoxynucleotidyl transferase dUTP nick-end labeling (TUNEL) assay. Cells were subjected to a TUNEL assay using an ApopTag InSitu apoptosis detection kit (cat. no. S7111; EMD Millipore, Billerica, MA, USA) according to the manufacturer's protocol. TUNEL-positive/apoptosis rate was presented as the percentage of total cells. Images were captured at $\mathrm{x} 40$ magnification with a fluorescence/phase contrast microscope (FSX100; Olympus Corp., Tokyo, Japan). Three independent experiments were performed.

Caspase 3 activity assay. Activity of caspase 3 was determined using a colorimetric caspase 3 assay kit (cat. no. ab39401) from Abcam (Cambridge, MA, USA). The assays were performed in 96-well plates by incubating $20 \mu \mathrm{l}$ cell lysate protein/sample in $70 \mu \mathrm{l}$ reaction buffer [1\% NP-40, $20 \mathrm{mM}$ Tris- $\mathrm{HCl}(\mathrm{pH} 7.5)$, $137 \mathrm{mM}$ Nad and $10 \%$ glycerol] containing $10 \mu \mathrm{l}$ caspase-3 substrate $(2 \mathrm{mM})$. Lysates were then incubated at $37^{\circ} \mathrm{C}$ for $6 \mathrm{~h}$, after which the samples were assayed using a spectrophotometer (cat. no. 1702525; Bio-Rad Laboratories, Hercules, CA, USA) at $405 \mathrm{~nm}$.

Reactive oxygen species (ROS) detection. ROS were measured using a dichlorofluorescin diacetate (DCFDA) Cellular Reactive Oxygen Species Detection Assay kit (cat. no. ab113851; Abcam, Cambridge, UK) according to the manufacturer's protocol. Cells were plated at $9 \times 10^{4}$ cells/well in black 96-well plates and incubated with $25 \mu \mathrm{M}$ DCFDA for $45 \mathrm{~min}$. Fluorescence was detected using a Victor3 1420 Multilabel Counter (PerkinElmer Instruments, Shanghai, China).

Phosphatidylinositol 3-kinase (PI3K) activity assay. PI3K activity was determined with a PI3K activity ELISA kit (cat. no. K-1000s; Echelon Biosciences Inc., Salt Lake City, UT, USA) according to the manufacturer's protocol (12). To functionally assess PI3K activity, PI3K was isolated by immunoprecipitation using a rabbit polyclonal anti-human PI3K antibody (cat. no. 06-195; EMD Millipore) to the p85 adapter subunit. The ability with which the co-precipitated catalytic p110 catalytic subunit was able to convert a standard PIP2 to PIP3 in a kinase reaction was subsequently assessed 
A

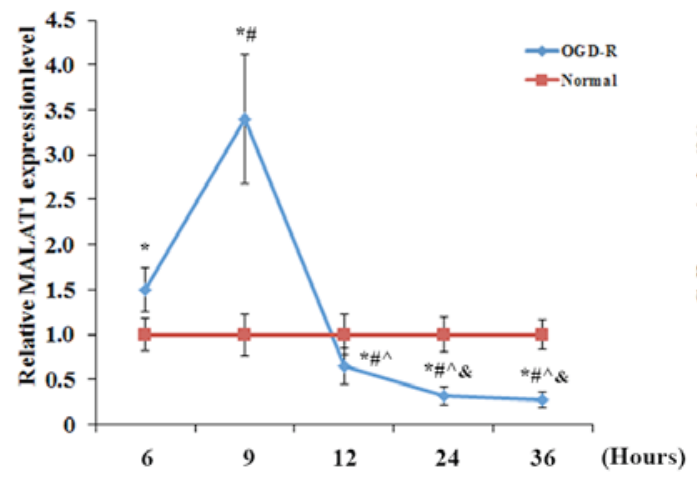

B

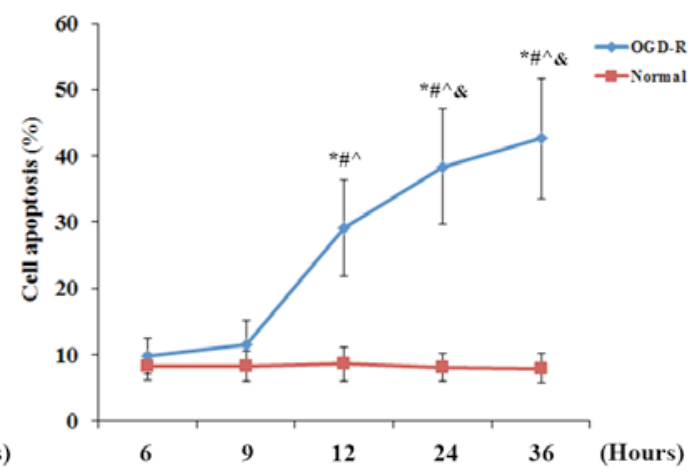

Figure 1. Expression of MALAT1 and apoptosis in brain vascular endothelial cells under OGD-R. Human brain microvascular endothelial cells were cultured under OGD-R. (A) The expression levels of MALAT1 were measured at 6, 9, 12, 24 and 36 h post-reoxygenation using reverse transcription-quantitative polymerase chain reaction and expressed as fold changes to that of the control cells under normal culture conditions (designated as 1 ); each group was normalized against RPLP0 mRNA expression levels as a reference gene. (B) The apoptotic rate of cells under OGD-R was measured at 6, 9, 12, 24 and 36 h post-reoxygenation with a microplate reader-based apoptosis detection kit. Cells under normal culture conditions were used as a control. Each experiment was performed in duplicate and repeated three times. ${ }^{*} \mathrm{P}<0.05$ vs. normal; ${ }^{\#} \mathrm{P}<0.05$ vs. $6 \mathrm{~h} ;{ }^{\wedge} \mathrm{P}<0.05$ vs. $9 \mathrm{~h}$; ${ }^{\&} \mathrm{P}<0.05$ vs. $12 \mathrm{~h}$. MALAT1, metastasis-associated lung adenoma transcript 1; OGD-R, oxygen-glucose deprivation and reoxygenation.

by measuring the PIP3, generated using an ELISA kit. Each experiment was repeated performed in duplicate, and was repeated three times.

Western blot analysis. Cells were lysed in a solution containing hypotonic buffer containing $0.5 \%$ Nonidet-P40 and a protease inhibitor cocktail (Sigma-Aldrich; Merck Millipore, Darmstadt, Germany) by sonication three times for $3 \mathrm{sec}$ on ice. Following centrifugation at 2,000 x $g$ for $15 \mathrm{~min}$ at $4^{\circ} \mathrm{C}$, the resulting supernatant was used for protein concentration determination by the Coomassie blue method (Thermo Fisher Scientific, Inc.) and for subsequent steps. From each sample, $5 \mu \mathrm{g}$ protein for each sample were separated by $10 \%$ SDS-polyacrylamide gel electrophoresis and were subsequently blotted onto a polyvinylidene difluoride microporous membrane (EMD Millipore). Membranes were blocked with $5 \%$ skim milk powder in Tris-buffered saline with tween 20 (TBS-T) for $2 \mathrm{~h}$ and incubated for $1 \mathrm{~h}$ at room temperature with rabbit polyclonal anti-human phosphorylated Akt (Ser 473) antibody (cat. no. sc-101629) or mouse monoclonal anti-human Akt antibody (cat. no. sc-81434) at a dilution of 1:1,000, and were subsequently washed three times in TBS-T (cat. no. SRE0031; Sigma-Aldrich; Merck Millipore) for $5 \mathrm{~min}$ and revealed using bovine anti-rabbit (sc-2370) or anti-mouse (sc-2371; all Santa Cruz Biotechnology, Inc., Dallas, TX, USA) secondary antibody (both 1:5,000) for $1 \mathrm{~h}$ at room temperature. Peroxidase was revealed with an enhanced chemiluminescence kit (GE Healthcare Life Sciences, Shanghai, China). Three independent experiments were performed.

Statistical analysis. Statistical analyses were performed using SPSS for Windows 19.0 (IBM SPSS, Armonk, NY, USA). All values were expressed as the mean \pm standard deviation. Comparisons of the means among multiple groups were performed with one-way analysis of variance followed by post-hoc pairwise comparisons using Tukey's tests. A two-tailed $\mathrm{P}<0.05$ was considered to indicate a statistically significant difference.

\section{Results}

MALAT1 inhibits OGD-R-induced apoptosis and caspase 3 activity, but not ROS production, in human brain vascular endothelial cells. In the present study, primary human brain microvascular endothelial cells exposed to OGD-R were employed as an in vitro model of cerebral vascular endothelial cell I/R injury, as previously described (1). As shown in Fig. 1, human brain microvascular endothelial cells were cultured under OGD-R, and the expression level of MALAT1 and apoptosis in the cells were measured at 6, 9, 12, 24 and $36 \mathrm{~h}$ post-reoxygenation. As shown in Fig. 1A, using cells under normal culture conditions for normalization, the expression level of MALAT1 in cells under OGD-R significantly increased $(\mathrm{P}<0.05)$ after reoxygenation, peaking at 1.5 -fold that of cells under normal culture conditions at $9 \mathrm{~h}$ post-reoxygenation, and significantly decreased $(\mathrm{P}<0.05)$ below the normal level, reaching 0.27 -fold that of cells under normal culture conditions at $36 \mathrm{~h}$ post-reoxygenation. The apoptosis rate of cells under normal culture conditions did not exhibit any significant change over time (Fig. 1B). Furthermore, no significant change was identified in the apoptotic rate of cells exposed to OGD-R at $6 \mathrm{~h}(9.8 \pm 2.7 \%)$ and $9 \mathrm{~h}(11.5 \pm 3.7 \%)$ post-reoxygenation, compared with the cells under normal culture conditions. However, the apoptotic rate of cells exposed to OGD-R markedly increased to $29.1 \pm 7.2 \%$ at $12 \mathrm{~h}$, reaching $42.6 \pm 9.1 \%$ at $36 \mathrm{~h}$ post-oxygenation (Fig. 1B). The contrasting trends of the expression levels of MALAT1 and cell apoptosis in human brain microvascular endothelial cells following OGD-R suggested that MALAT1 may inhibit OGD-R-induced apoptosis in brain vascular endothelial cells.

To explore the effect of MALAT1 on OGD-R-induced apoptosis of brain vascular endothelial cells, human brain microvascular endothelial cells were transduced with human MALAT1 lentivirus or human MALAT1 siRNA lentivirus to overexpress or knockdown MALAT1, respectively. Cells transduced with the blank control lentivirus (VC) or the scrambled siRNA lentivirus (SC) were used as controls. To investigate 
A

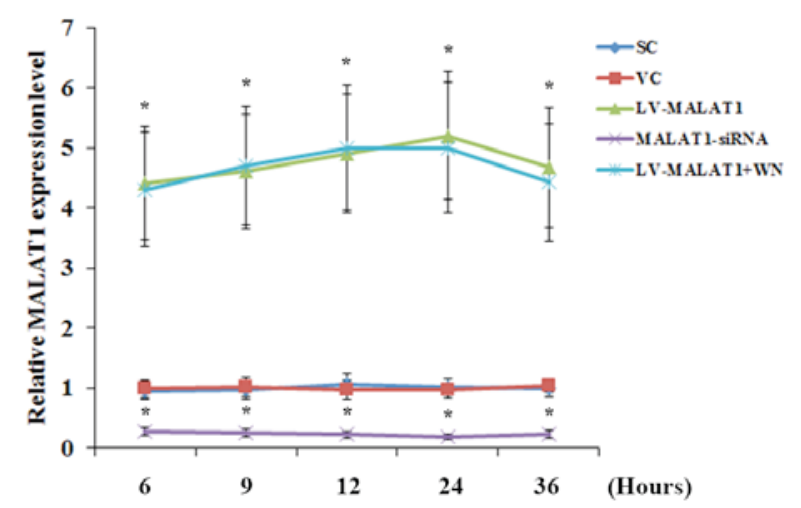

B

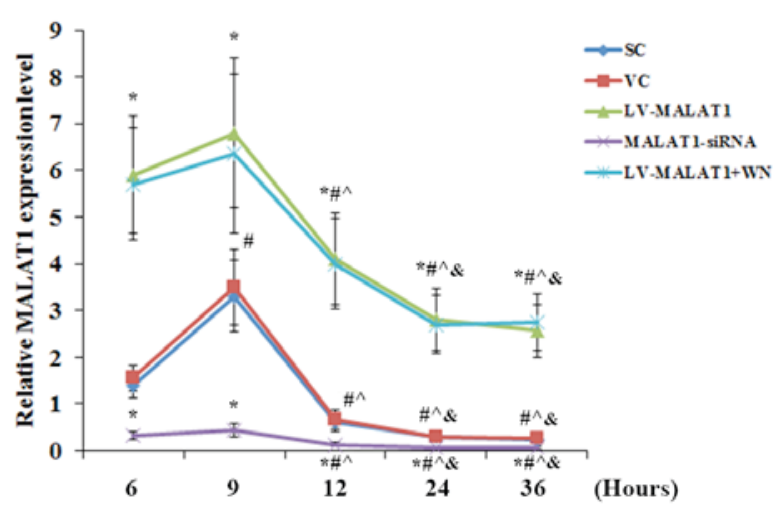

Figure 2. Overexpression and knockdown of MALAT1 in brain vascular endothelial cells in the presence or absence of OGD-R. Human brain microvascular endothelial cells were transduced with human LV-MALAT1 or human MALAT1-siRNA to overexpress or knockdown MALAT1, respectively. Cells transduced with VC or SC were used as controls. Cells transduced with human LV-MALAT1 were also treated with a phosphatidylinositol 3-kinase inhibitor, Wortmannin $(50 \mu \mathrm{M})$, during reoxygenation. The expression level of MALAT1 (A) in the absence or (B) presence of OGD-R (specifically, at 6, 9, 12, 24 and $36 \mathrm{~h}$ post-reoxygenation) was measured via reverse transcription-quantitative polymerase chain reaction and expressed as fold changes to that of the control cells under normal culture conditions (designated as 1); each group was normalized against RPLP0 mRNA expression levels as a reference gene. Each experiment was performed in duplicate and repeated three times. ${ }^{*} \mathrm{P}<0.05$ vs. SC and VC; ${ }^{\#} \mathrm{P}<0.05$ vs. $6 \mathrm{~h}$; ${ }^{\wedge} \mathrm{P}<0.05$ vs. $9 \mathrm{~h}$; ${ }^{\&} \mathrm{P}<0.05$ vs. $12 \mathrm{~h}$. MALAT1, metastasis-associated lung adenoma transcript 1; OGD-R, oxygen-glucose deprivation and reoxygenation; LV-MALAT1, MALAT1 lentivirus; MALAT1-siRNA, MALAT1 siRNA lentivirus; VC, blank control lentivirus; SC, scrambled siRNA lentivirus; LV-MALAT1+WN, MALAT1 lentivirus-Wortmannin.

A

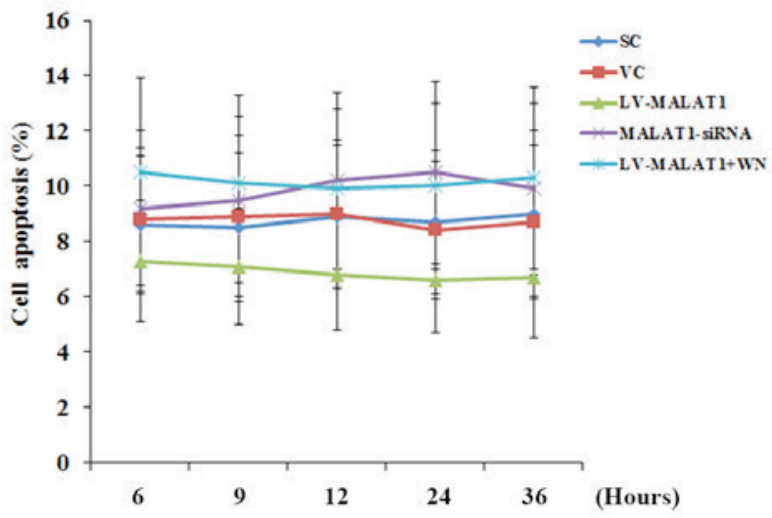

$\mathbf{B}$

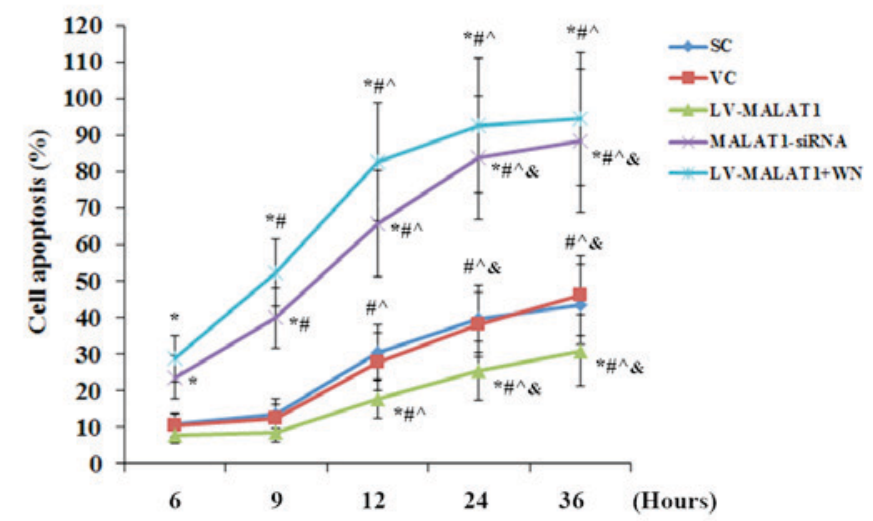

Figure 3. Apoptosis in brain vascular endothelial cells with overexpression or knockdown of MALAT1 in the presence or absence of OGD-R. Human brain microvascular endothelial cells were transduced with human LV-MALAT1 or MALAT1-siRNA to overexpress or knockdown MALAT1, respectively. Cells transduced with VC or SC were used as controls. Cells transduced with human MALAT1 lentivirus were also treated with phosphatidylinositol 3-kinase inhibitor, Wortmannin $(50 \mu \mathrm{M})$, during reoxygenation. The apoptotic rate of cells (A) in the absence or (B) the presence of OGD-R (specifically, at 6, 9, 12,24 and $36 \mathrm{~h}$ post-reoxygenation) was measured with a microplate reader-based apoptosis detection kit. Each experiment was performed in duplicate and repeated three times. ${ }^{*} \mathrm{P}<0.05$ vs. SC and VC; ${ }^{~} \mathrm{P}<0.05$ vs. $6 \mathrm{~h} ;{ }^{\wedge} \mathrm{P}<0.05$ vs. $9 \mathrm{~h} ;{ }^{\&} \mathrm{P}<0.05$ vs. $12 \mathrm{~h}$. MALAT1, metastasis-associated lung adenoma transcript 1 ; OGD-R, oxygen-glucose deprivation and reoxygenation; LV-MALAT1, MALAT1 lentivirus; MALAT1-siRNA, MALAT1 siRNA lentivirus; VC, blank control lentivirus; SC, scrambled siRNA lentivirus; LV-MALAT1+WN, MALAT1 lentivirus-Wortmannin.

the potential role of PI3K/Akt survival signaling in the effects induced by MALAT1, cells overexpressing MALAT1 were treated with a PI3K inhibitor, Wortmannin. As shown in Fig. 2, the VC and SC controls exposed to OGD-R exhibited similar changes in the expression level of MALAT1 as nontransduced cells in Fig. 1A, indicating that transduction of the VC or SC did not significantly affect the expression level of MALAT1. In the presence or absence of OGD-R, lentiviral transduction of MALAT1 and MALAT1-siRNA resulted in a significant increase and decrease (both $\mathrm{P}<0.05$ ) in the expression levels of MALAT1, when compared with the controls, respectively (Fig. 2). No significant change was detected in the expression levels of MALAT1 over time in either of the experimental groups in the absence of OGD-R (Fig. 2A). In the presence of OGD-R, all experimental groups exhibited a significant increase in MALAT1 expression levels by $9 \mathrm{~h}$ post-reoxygenation, followed by a continuous decrease $(\mathrm{P}<0.05)$ (Fig. 2B). Inhibition of PI3K signaling by Wortmannin showed no significant effect on the expression level of MALAT1 in the presence or absence of OGD-R (Fig. 2).

As shown in Fig. 3A, in the absence of OGD-R, there was no significant change detected in the cell apoptosis rate over time in each experimental group, compared with the controls. Similarly, the overexpression and knockdown of MALAT1 and inhibition of PI3K signaling by Wortmannin also exhibited no significant effect on cell apoptosis. These results 
A

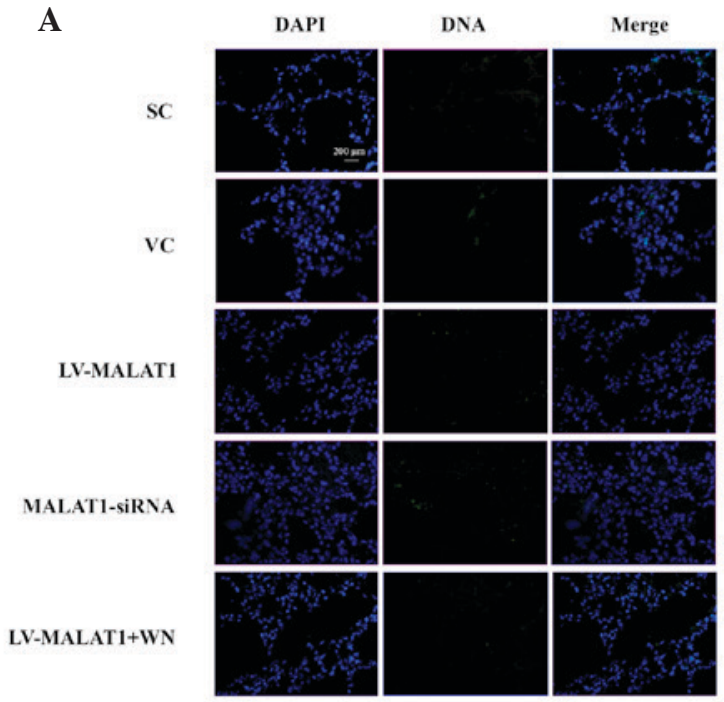

B

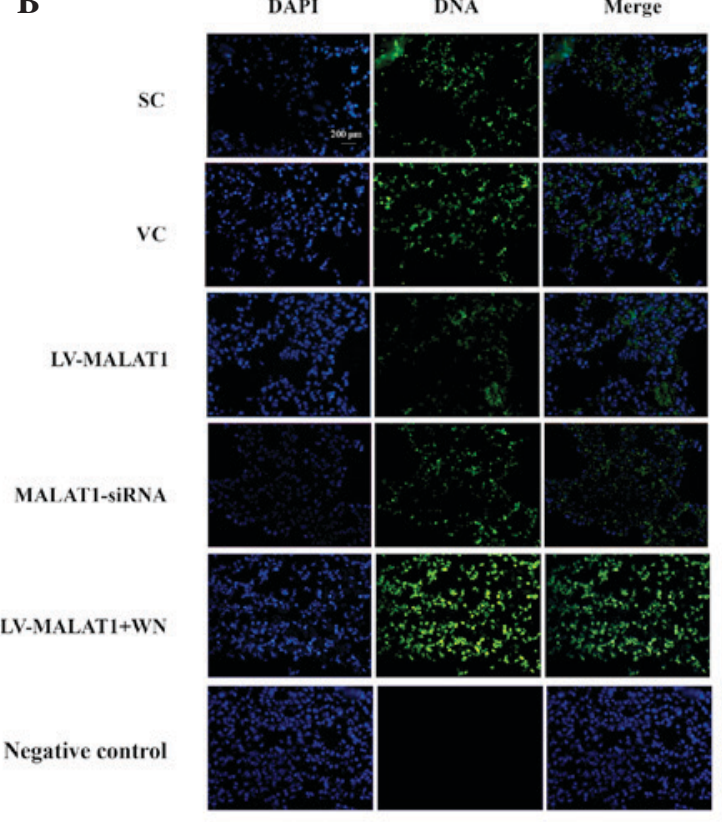

C

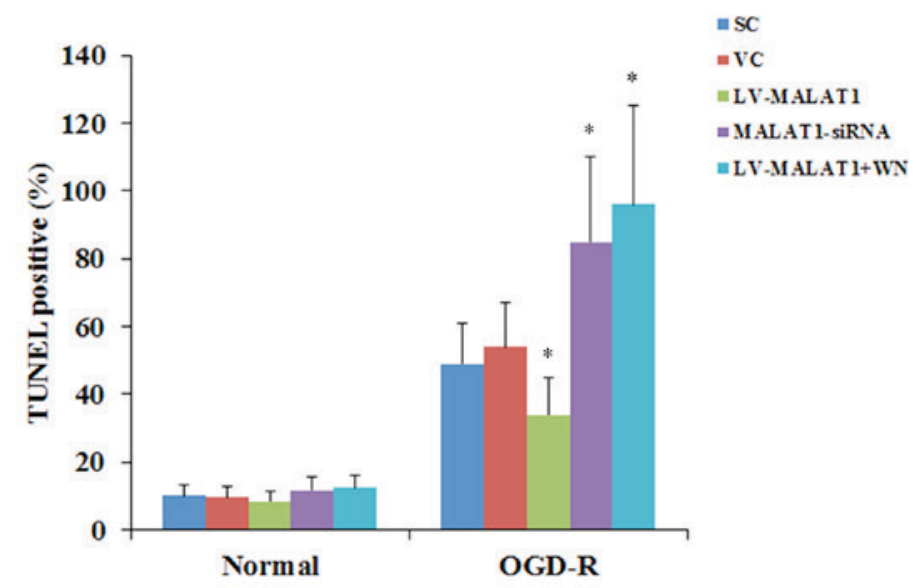

Figure 4. TUNEL staining in brain vascular endothelial cells with overexpression or knockdown of MALAT1 in the presence or absence of OGD-R. Human brain microvascular endothelial cells were transduced with human LV-MALAT1 or MALAT1-siRNA to overexpress or knockdown MALAT1, respectively. Cells transduced with VC or SC lentivirus were used as controls. Cells transduced with human LV-MALAT1 were also treated with phosphatidylinositol 3-kinase inhibitor, Wortmannin $(50 \mu \mathrm{M})$, during reoxygenation. The apoptotic rate of cells (A) in the absence or (B) presence of OGD-R at $36 \mathrm{~h}$ post-reoxygenation was measured with a TUNEL staining kit. The images present cell nuclei stained by DAPI (blue) and DNA fragmentation (green). (C) The TUNEL-positive/apoptosis rate is shown as the percentage of total cells in histograms. Each experiment was repeated three times. $\mathrm{P}<0.05$ vs. SC and VC. TUNEL, terminal deoxynucleotidyl transferase dUTP nick-end labeling; MALAT1, metastasis-associated lung adenoma transcript 1; OGD-R, oxygen-glucose deprivation and reoxygenation; LV-MALAT1, MALAT1 lentivirus; MALAT1-siRNA, MALAT1 siRNA lentivirus; VC, blank control lentivirus; SC, scrambled siRNA lentivirus; LV-MALAT1+WN, MALAT1 lentivirus-Wortmannin.

suggested that MALAT1 had no significant effect on human brain vascular endothelial cell apoptosis under normal conditions. In the presence of OGD-R, however, overexpression of MALAT1 significantly decreased cell apoptosis compared with the controls $(\mathrm{P}<0.05)$; this effect was abolished by Wortmannin (Fig. 3B). However, knockdown of MALAT1 significantly increased cell apoptosis compared with cells transfected with the blank control lentivirus $(\mathrm{P}<0.05)$, up to $\sim 90 \%$ at 36 h post-reoxygenation (Fig. 3B). TUNEL staining at $36 \mathrm{~h}$ post-reoxygenation corroborated these findings (Fig. 4).
Consistent with the cell apoptosis data, in the absence of OGD-R, there was no significant change identified between the groups or over time in the activity of caspase 3 (Fig. 5A), a critical caspase in the apoptotic pathway (14). In the presence of OGD-R, however, overexpression of MALAT1 significantly decreased caspase 3 activity compared with the controls $(\mathrm{P}<0.05)$. This effect was abolished by Wortmannin; however, knockdown of MALAT1 markedly increased the caspase 3 activity compared with the controls (Fig. 5B).

OGD-R has been shown to induce cellular overproduction of ROS, which leads to cell apoptosis (15-18). Therefore, 
A

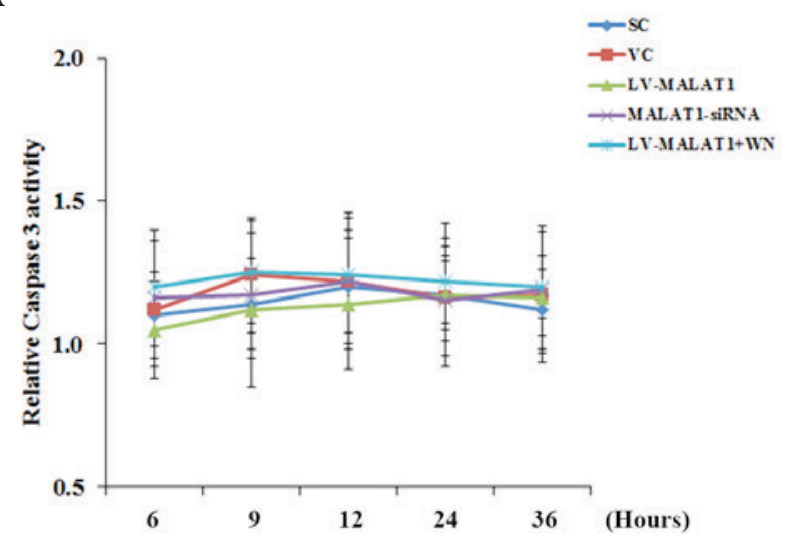

B

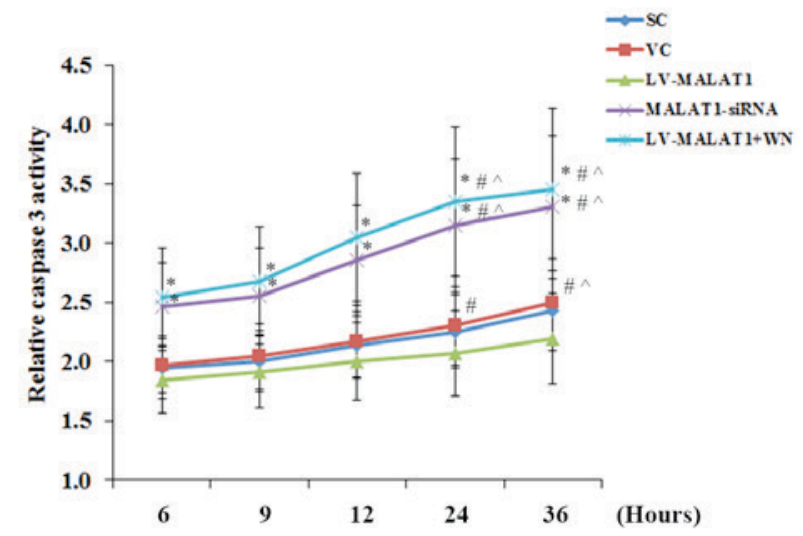

Figure 5. Caspase 3 activity in brain vascular endothelial cells with overexpression or knockdown of MALAT1 in the presence or absence of OGD-R. Human brain microvascular endothelial cells were transduced with human LV-MALAT1 MALAT1 or MALAT1-siRNA to overexpress or knockdown MALAT1, respectively. Cells transduced with VC or SC lentivirus were used as controls. Cells transduced with human LV-MALAT1 were also treated with phosphatidylinositol 3-kinase inhibitor, Wortmannin $(50 \mu \mathrm{M})$, during reoxygenation. The caspase 3 activity in cells (A) in the absence or (B) presence of OGD-R (specifically, at 6,9,12,24 and $36 \mathrm{~h}$ post-reoxygenation) was measured with a colorimetric caspase 3 assay kit and and expressed as fold changes to that of cells under normal culture conditions (designated as 1). Each experiment was performed in duplicate and repeated three times. "P $\mathrm{P}<0.05$ vs. SC and VC; ${ }^{*} \mathrm{P}<0.05$ vs. $6 \mathrm{~h}$; ${ }^{\wedge} \mathrm{P}<0.05$ vs. $9 \mathrm{~h}$; ${ }^{\circledR} \mathrm{P}<0.05$ vs. $12 \mathrm{~h}$. MALAT1, metastasis-associated lung adenoma transcript 1; OGD-R, oxygen-glucose deprivation and reoxygenation; LV-MALAT1, MALAT1 lentivirus; MALAT1-siRNA, MALAT1 siRNA lentivirus; VC, blank control lentivirus; SC, scrambled siRNA lentivirus; LV-MALAT1+WN, MALAT1 lentivirus-Wortmannin.

A

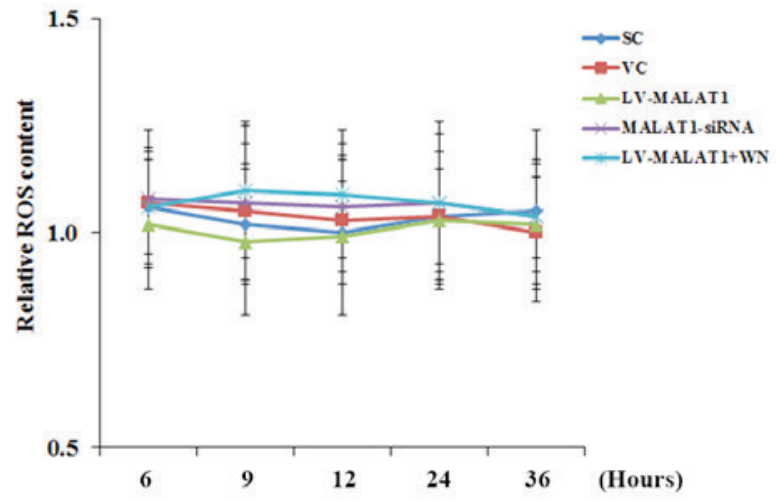

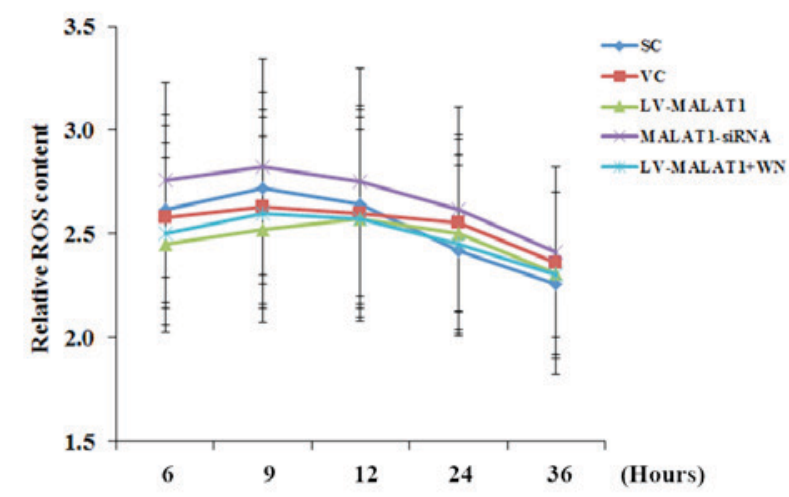

Figure 6. ROS production in brain vascular endothelial cells with overexpression or knockdown of MALAT1 in the presence or absence of OGD-R. Human brain microvascular endothelial cells were transduced with human LV-MALAT1 or MALAT1-siRNA to overexpress or knockdown MALAT1, respectively. Cells transduced with VC or SC lentivirus were used as controls. Cells transduced with human LV-MALAT1 were also treated with phosphatidylinositol 3-kinase inhibitor, Wortmannin $(50 \mu \mathrm{M})$, during reoxygenation. ROS production in cells (A) in the absence or (B) presence of OGD-R (specifically, at 6, 9, 12, 24 and $36 \mathrm{~h}$ post-reoxygenation) was measured with a fluorescence cellular ROS detection assay kit and expressed as fold changes to that of the control cells under normal culture conditions (designated as 1). Each experiment was performed in duplicate and repeated three times. ${ }^{*} \mathrm{P}<0.05$ vs. SC and VC; ${ }^{*} \mathrm{P}<0.05$ vs. $6 \mathrm{~h} ;{ }^{\wedge} \mathrm{P}<0.05$ vs. $9 \mathrm{~h}$; ${ }^{\star} \mathrm{P}<0.05$ vs. $12 \mathrm{~h}$. ROS, reactive oxygen species; MALAT1, metastasis-associated lung adenoma transcript 1; OGD-R, oxygen-glucose deprivation and reoxygenation; LV-MALAT1, MALAT1 lentivirus; MALAT1-siRNA, MALAT1 siRNA lentivirus; VC, blank control lentivirus; SC, scrambled siRNA lentivirus; LV-MALAT1+WN, MALAT1 lentivirus-Wortmannin.

the effect of MALAT1 on ROS production in human brain microvascular endothelial cells in the presence and absence of OGD-R was investigated. As shown in Fig. 6, in the absence of OGD-R, the ROS content in all experimental groups remained similar to the levels exhibited by cells under normal culture conditions (designated as 1) over time. However, in the presence of OGD-R, the ROS content in all experimental groups increases $\sim 2.5$-fold, compared with the cells under normal culture conditions, with no significant differences identified between the groups or over time.

These findings suggested that MALAT1 may protect human brain vascular endothelial cells from OGD-R-induced apoptosis, not by reducing OGD-R-induced ROS overproduction, but by a PI3K-dependent survival mechanism. Therefore, 
A

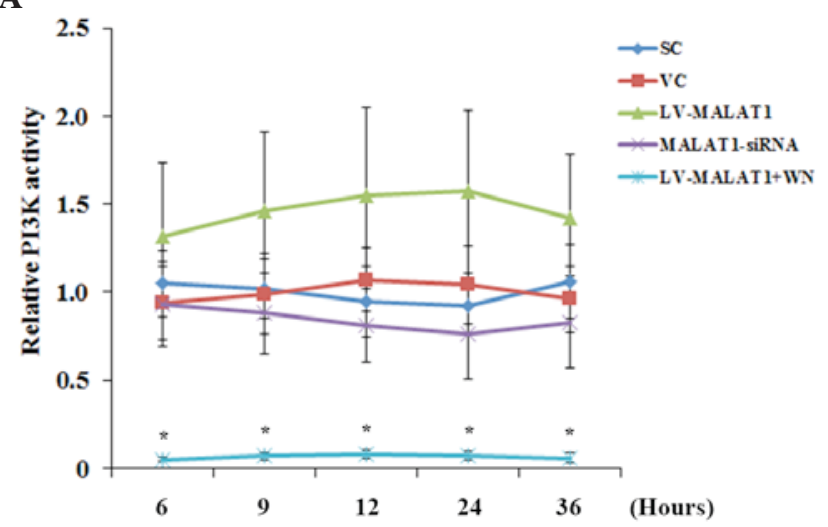

B

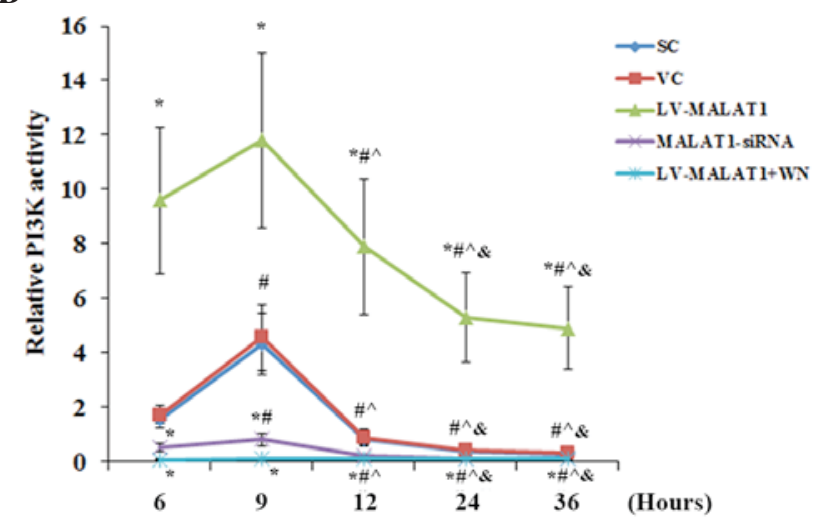

Figure 7. PI3K activity in brain vascular endothelial cells with overexpression or knockdown of MALAT1 in the presence or absence of OGD-R. Human brain microvascular endothelial cells were transduced with human LV-MALAT1) or MALAT1-siRNA to overexpress or knockdown MALAT1, respectively. Cells transduced with VC or SC were used as controls. Cells transduced with human LV-MALAT1 were also treated with PI3K inhibitor, Wortmannin (50 $\mu \mathrm{M})$, during reoxygenation. The PI3K activity in cells (A) in the absence or (B) the presence of OGD-R (specifically, at 6, 9, 12, 24 and 36 h post-reoxygenation) was measured with a PI3K activity ELISA kit and expressed as fold changes to that of the control cells under normal culture conditions (designated as 1$)$. Each experiment was performed in duplicate and repeated three times. ${ }^{*} \mathrm{P}<0.05$ vs. SC and VC; ${ }^{\#} \mathrm{P}<0.05 \mathrm{vs} .6 \mathrm{~h} ;{ }^{\wedge} \mathrm{P}<0.05 \mathrm{vs} .9 \mathrm{~h}$; ${ }^{\&} \mathrm{P}<0.05 \mathrm{vs}$. $12 \mathrm{~h}$. PI3K, phosphatidylinositol 3-kinase; MALAT1, metastasis-associated lung adenoma transcript 1; OGD-R, oxygen-glucose deprivation and reoxygenation; LV-MALAT1, MALAT1 lentivirus; MALAT1-siRNA, MALAT1 siRNA lentivirus; VC, blank control lentivirus; SC, scrambled siRNA lentivirus; LV-MALAT1+WN, MALAT1 lentivirus-Wortmannin.

the effect of MALAT1 on PI3K/Akt survival signaling, which reportedly protects cerebral endothelial cells from hypoxia/reoxygenation-induced apoptosis (17), was subsequently examined in human brain microvascular endothelial cells in the presence and absence of OGD-R.

Effect of MALAT1 on PI3K/Akt survival signaling in human brain microvascular endothelial cells. Overexpression and knockdown of MALAT1 had no significant effect on PI3K activity in the absence of OGD-R compared with the controls, except when Wortmannin completely abolished PI3K activity (Fig. 7A). There was no significant change detected in PI3K activity over time in each experimental group. However, in the presence of OGD-R, overexpression of MALAT1 markedly increased PI3K activity in human brain microvascular endothelial cells compared with the controls; this effect was completely abolished by Wortmannin (Fig. 7B). Knockdown of MALAT1 significantly decreased $(\mathrm{P}<0.05) \mathrm{PI} 3 \mathrm{~K}$ activity compared with the controls (Fig. 7B). Similar data trends were observed with the activation of the phosphorylation of Akt at serine 473 (17) in human brain microvascular endothelial cells in the presence and absence of OGD-R, respectively (Fig. 8). These findings indicated that MALAT1 may protect human brain vascular endothelial cells from OGD-R-induced apoptosis via the PI3K/Akt survival pathway.

\section{Discussion}

Cerebral I/R injury is a common cause of debilitating complications and mortality associated with stroke and traumatic brain injury (1), often leading to brain vascular dysfunction characterized by endothelial cell injury or apoptosis (2). A recent study suggested that lncRNAs may function as a class of novel master regulators in cerebrovascular endothelial pathologies after ischemic stroke (8). MALAT1, a lncRNA which was initially shown to control tumor metastasis and cancer cell survival (9), has been demonstrated to have a role in endothelial cell function and dysfunction (9-11). In the present study, through the use of primary human brain microvascular endothelial cells under an in vitro I/ $\mathrm{R}$ injury setting (1), it was observed that MALAT1 inhibits OGD-R-induced human brain vascular endothelial cell apoptosis.

OGD-R has been used as an in vitro model of cerebral vascular endothelial cell I/R injury in previous studies and has been shown to cause significant (25-40\%) endothelial cell apoptosis $(17,18)$. In the present study it was observed that OGD-R induced $\sim 40 \%$ endothelial cell apoptosis. The expression level of MALAT1 and the apoptosis rate in human brain microvascular endothelial cells under OGD-R exhibited contrasting trends after reoxygenation. Notably, OGD-R induced a marked increase in the expression levels of MALAT1 by $9 \mathrm{~h}$ post-reoxygenation, followed by a continuous decrease. Conversely, the cell apoptosis rate remained similar to that of cells under normal culture conditions by $9 \mathrm{~h}$ post-reoxygenation, followed by a continuous increase. Subsequently, overexpression and knockdown experiments revealed that, in cells exposed to OGD-R, overexpression of MALAT1 significantly decreased cell apoptosis, and knockdown of MALAT1 markedly increased cell apoptosis by up to $\sim 90 \%$. The increase of MALAT1 by $9 \mathrm{~h}$ post-oxygenation, which corresponded to inhibited cell apoptosis at that time point, may be a cellular physiological response to protect brain vascular endothelial cells against OGD-R-induced apoptosis. These findings suggest that MALAT1 may be a potent natural protector of brain vascular endothelium against OGD-R insult in vitro, and thereby $\mathrm{I} / \mathrm{R}$ injury in vivo. Therefore, MALAT1 may be a potential novel therapeutic target for cerebral I/R injury in stroke and traumatic brain injury, although future in vivo studies are required to verify its application potential. In addition, it is unclear why the expression level of MALAT1 
A

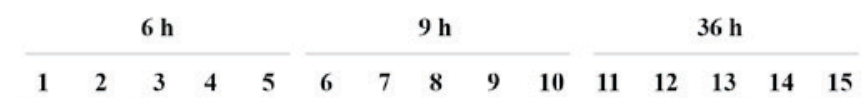

p-Akt

Total Akt

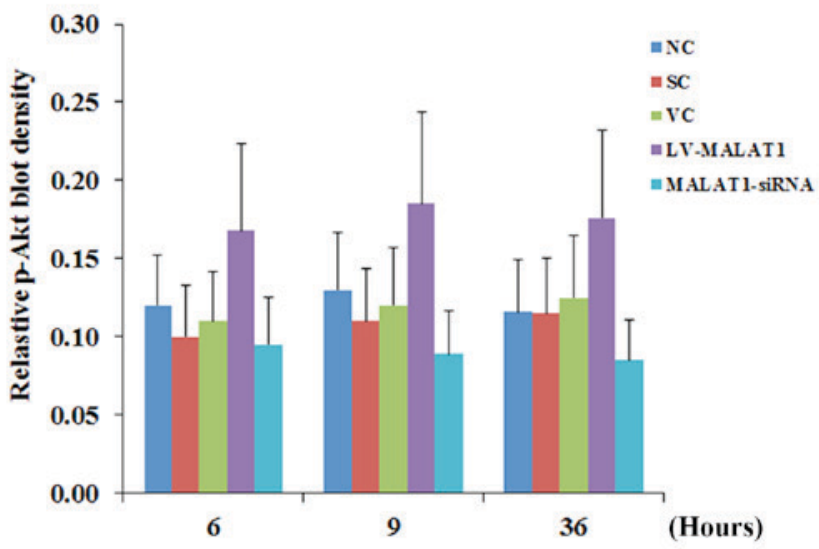

$\mathbf{B}$
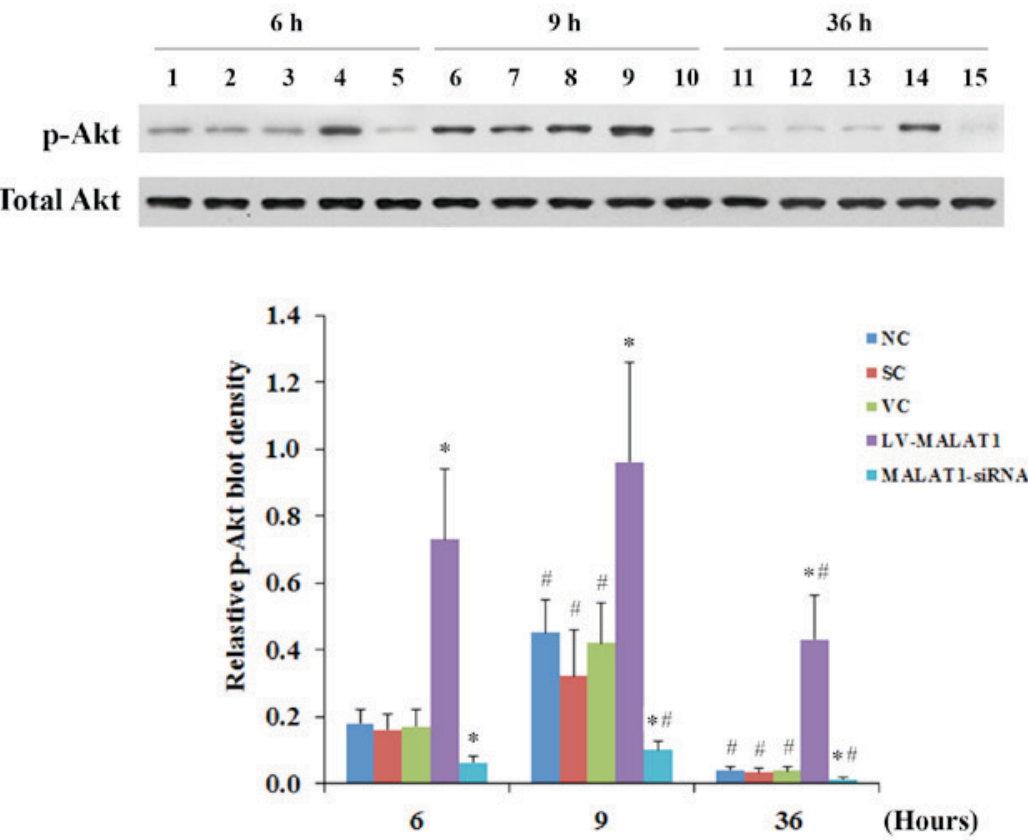

Figure 8. p-Akt levels in brain vascular endothelial cells with overexpression or knockdown of MALAT1 in the presence or absence of OGD-R. Human brain microvascular endothelial cells were transduced with human MALAT1 LV-MALAT1 or MALAT1-siRNA to overexpress or knockdown MALAT1, respectively. Cells transduced with VC or SC were used as controls. Cells transduced with human LV-MALAT1 were also treated with phosphatidylinositol 3-kinase inhibitor, Wortmannin $(50 \mu \mathrm{M})$, during reoxygenation. Levels of total Akt and p-Akt at ser473 were determined by western blot analyses (A) in the absence or (B) the presence of OGD-R (specifically, at 6, 9, 12, 24 and $36 \mathrm{~h}$ post-reoxygenation). Lanes 1, 6 and 11: NC; lanes 2, 7 and 12: VC; lanes 3, 8 and 13: SC; lanes 4, 9 and 14: LV-MALAT1; lanes 5, 10 and 15: MALAT1-siRNA. Lanes 1-5: $6 \mathrm{~h}$ post-reoxygenation; lanes 6-10: $9 \mathrm{~h}$ post-reoxygenation; lanes 11-15: $36 \mathrm{~h}$ post-reoxygenation. The total Akt level was not significantly altered by OGD-R. Density of the p-Akt (ser473) blot was normalized against that of total Akt to obtain a relative p-Akt blot density. Each experiment was repeated three times. " $\mathrm{P}<0.05$ vs. NC, SC and VC; ${ }^{*} \mathrm{P}<0.05$ vs. 6 h. p-Akt, phosphorylated Akt; MALAT1, metastasis-associated lung adenoma transcript 1; OGD-R, oxygen-glucose deprivation and reoxygenation; LV-MALAT1, MALAT1 lentivirus; MALAT1-siRNA, MALAT1 siRNA lentivirus; NC, normal control cells; VC, blank control lentivirus; SC, scrambled siRNA lentivirus; LV-MALAT1+WN, MALAT1 lentivirus-Wortmannin; ser473, serine 473.

markedly decreased after peaking at $9 \mathrm{~h}$ post-reoxygenation. This trend remained following the overexpression of MALAT1, which significantly elevated the overall expression level of MALAT1 above those of the controls. This may be a compensatory cellular response to accelerate the degradation of MALAT1 after an acute elevation of its expression. Further 
study is required to elucidate the underlying mechanisms and physiological significance.

The activity of caspase 3, which is a highly active caspase that is present in apoptotic cells (13), corroborated the findings from the present study that indicated that MALAT1 inhibits OGD-R-induced human brain microvascular endothelial cell apoptosis. It has been reported that OGD-R induces cellular overproduction of ROS, which leads to cell apoptosis (15-19). In the present study, it was revealed that OGD-R significantly increased cellular ROS content. However, unlike caspase 3 activity, ROS content was not significantly altered by the expression level of MALAT1, thereby excluding the possibility that MALAT1 protected human brain vascular endothelial cells from OGD-R-induced apoptosis by regulating OGD-R-induced ROS overproduction. Conversely, in cells exposed to OGD-R, the Wortmannin PI3K inhibitor did not alter the expression level of MALAT1; however, the apoptosis protective effect of MALAT1 was abolished, suggesting that MALAT1 may protect human brain vascular endothelial cells from OGD-R-induced apoptosis via a PI3K-dependent mechanism. This was subsequently corroborated by PI3K activity assay results and the phosphorylated levels of Akt, indicating that the protective effect of MALAT1 against OGD-R-induced apoptosis in human brain vascular endothelial cells may be mediated through the PI3K/Akt survival signaling pathway. These findings are in agreement with previous studies that have reported that MALAT1 activates the PI3K/Akt pathway $(20,21)$, which reportedly protects human cerebral endothelial cells from hypoxia/reoxygenation-induced oxidative stress and apoptosis (18). In addition, it has been shown that PI3K/Akt activation is able to suppress cell apoptosis by inhibiting caspase activation (22), which supports the findings from the present study that PI3K activity and caspase 3 activity in human brain microvascular endothelial cells exposed to OGD-R exhibited contrasting trends after reoxygenation. These findings suggest that activating the PI3K/Akt survival pathway in response to apoptotic stress may be a major mechanism underlying the protective effects of MALAT1 on human brain vascular endothelial cells under OGD-R.

Notably, MALAT1 did not significantly alter PI3K signaling activity in cells under normal culture conditions, only in cells exposed to OGD-R, which suggests that the presence of in vitro OGD-R insult or in vivo $\mathrm{I} / \mathrm{R}$ injury is required to activate MALAT1/PI3K/Akt signaling in human brain vascular endothelial cells. How MALAT1 activates the PI3K/Akt pathway in human brain vascular endothelial cells exposed to OGD-R remains unclear. Previous studies have shown that MALAT1 is able to interact with polycomb 2 and thereby regulate histone modifications to control cellular proliferation $(23,24)$. In addition, it has been reported that MALAT1 interacts with serine-/arginine-rich proteins to regulate the subcellular localization of proteins that regulate splicing (24). A recent study revealed that MALAT1 is critical for maintaining the blood-brain/blood-tumor barrier, which is characterized by the presence of tight junctions between brain capillary endothelial cells that restrict paracellular diffusion (25). This suggests that MALAT1 may regulate cerebral vascular endothelial barrier functions. It is not yet clear whether these mechanisms are associated with the activation of MALAT1/PI3K/Akt signaling in human brain vascular endothelial cells under OGD-R in vitro or $\mathrm{I} / \mathrm{R}$ in vivo.

In conclusion, to the best of our knowledge, the present study was the first to indicate that lncRNA MALAT1 protects human brain vascular endothelial cells from OGD-R-induced apoptosis via a PI3K-dependent mechanism, suggesting that MALAT1 may be a potential novel therapeutic target for cerebral I/R injury. Further study is required in order to elucidate the underlying mechanisms.

\section{Acknowledgements}

This work was supported by the Hunan Provincial Bureau of Science and Technology, Changsha, China (grant no. 20151022).

\section{References}

1. Alluri H, Anasooya Shaji C, Davis ML and Tharakan B: Oxygen-glucose deprivation and reoxygenation as an in vitro ischemia-reperfusion injury model for studying blood-brain barrier dysfunction. J Vis Exp: e52699, 2015.

2. Zhang Y, Zhang X, Park TS and Gidday JM: Cerebral endothelial cell apoptosis after ischemia-reperfusion: Role of PARP activation and AIF translocation. J Cereb Blood Flow Metab 25: 868-877, 2005.

3. Vausort M, Wagner DR and Devaux Y: Long noncoding RNAs in patients with acute myocardial infarction. Circ Res 115: 668-677, 2014.

4. Carninci P, Kasukawa T, Katayama S, Gough J, Frith MC, Maeda N, Oyama R, Ravasi T, Lenhard B, Wells C, et al: The transcriptional landscape of the mammalian genome. Science 309: 1559-1563, 2005.

5. Kapranov P, Cheng J, Dike S, Nix DA, Duttagupta R, Willingham AT, Stadler PF, Hertel J, Hackermüller J, Hofacker IL, et al: RNA maps reveal new RNA classes and a possible function for pervasive transcription. Science 316: 1484-1488, 2007.

6. Mercer TR and Mattick JS: Structure and function of long noncoding RNAs in epigenetic regulation. Nat Struct Mol Biol 20: 300-307, 2013.

7. $\mathrm{Hu}$ W, Alvarez-Dominguez JR and Lodish HF: Regulation of mammalian cell differentiation by long non-coding RNAs. EMBO Rep 13: 971-983, 2012.

8. Zhang J, Yuan L, Zhang X, Hamblin MH, Zhu T, Meng F, Li Y, Chen YE and Yin KJ: Altered long non-coding RNA transcriptomic profiles in brain microvascular endothelium after cerebral ischemia. Exp Neurol 277: 162-170, 2016.

9. Puthanveetil P, Chen S, Feng B, Gautam A and Chakrabarti S: Long non-coding RNA MALAT1 regulates hyperglycaemia induced inflammatory process in the endothelial cells. J Cell Mol Med 19: 1418-1425, 2015.

10. Michalik KM, You X, Manavski Y, Doddaballapur A, Zörnig M, Braun T, John D, Ponomareva Y, Chen W, Uchida S, et al: Long noncoding RNA MALAT1 regulates endothelial cell function and vessel growth. Circ Res 114: 1389-1397, 2014.

11. Thum T and Fiedler J: LINCing MALAT1 and angiogenesis. Circ Res 114: 1366-1368, 2014.

12. Mysiorek C, Culot M, Dehouck L, Derudas B, Staels B, Bordet R, Cecchelli R, Fenart L and Berezowski V: Peroxisome-proliferator-activated receptor-alpha activation protects brain capillary endothelial cells from oxygen-glucose deprivation-induced hyperpermeability in the blood-brain barrier. Curr Neurovasc Res 6: 181-193, 2009.

13. Livak KJ and Schmittgen TD: Analysis of relative gene expression data using real-time quantitative PCR and the 2(-DeltaDelta C(T)) method. Methods 25: 402-408, 2001. 
14. Woo M, Hakem R, Soengas MS, Duncan GS, Shahinian A, Kägi D, Hakem A, McCurrach M, Khoo W, Kaufman SA, et al: Essential contribution of caspase $3 / \mathrm{CPP} 32$ to apoptosis and its associated nuclear changes. Genes Dev 12: 806-819, 1998

15. Gao XY, Huang JO, Hu YF, Gu Y, Zhu SZ, Huang KB, Chen JY and Pan SY: Combination of mild hypothermia with neuroprotectants has greater neuroprotective effects during oxygen-glucose deprivation and reoxygenation-mediated neuronal injury. Sci Rep 4: 7091, 2014.

16. Abramov AY, Scorziello A and Duchen MR: Three distinct mechanisms generate oxygen free radicals in neurons and contribute to cell death during anoxia and deoxygenation. J Neurosci 27: 1129-1138, 2007.

17. Zhang Y, Park TS and Gidday JM: Hypoxic preconditioning protects human brain endothelium from ischemic apoptosis by Akt-dependent survivin activation. Am J Physiol Heart Circ Physiol 292: H2573-H2581, 2007.

18. Wang J, Chen Y, Yang Y, Xiao X, Chen S, Zhang C, Jacobs B, Zhao B, Bihl J and Chen Y: Endothelial progenitor cells and neural progenitor cells synergistically protect cerebral endothelial cells from Hypoxia/reoxygenation-induced injury via activating the PI3K/Akt pathway. Mol Brain 9: 12, 2016.

19. Wang J, Zhang Y, Liu X, Ma J, Liu P, Hu C and Zhang G: Annexin A5 inhibits diffuse large B-cell lymphoma cell invasion and chemoresistance through phosphatidylinositol 3-kinase signaling. Oncol Rep 32: 2557-2563, 2014.
20. Xu S, Sui S, Zhang J, Bai N, Shi Q, Zhang G, Gao S, You Z, Zhan C, Liu F and Pang D: Downregulation of long noncoding RNA MALAT1 induces epithelial-to-mesenchymal transition via the PI3K-AKT pathway in breast cancer. Int J Clin Exp Pathol 8: 4881-4891, 2015.

21. Dong Y, Liang G, Yuan B, Yang C, Gao R and Zhou X: MALAT1 promotes the proliferation and metastasis of osteosarcoma cells by activating the PI3K/Akt pathway. Tumour Biol 36: 1477-1486, 2015.

22. Chen L, Wang J, Wang B, Yang J, Gong Z, Zhao X, Zhang C and Du K: MiR-126 inhibits vascular endothelial cell apoptosis through targeting PI3K/Akt signaling. Ann Hematol 95: 365-374, 2016.

23. Yang L, Lin C,Liu W,Zhang J, Ohgi KA, Grinstein JD, Dorrestein PC and Rosenfeld MG: ncRNA- and Pc2 methylation-dependent gene relocation between nuclear structures mediates gene activation programs. Cell 147: 773-788, 2011.

24. Tripathi V, Ellis JD, Shen Z, Song DY, Pan Q, Watt AT, Freier SM, Bennett CF, Sharma A, Bubulya PA, et al: The nuclear-retained noncoding RNA MALAT1 regulates alternative splicing by modulating SR splicing factor phosphorylation. Mol Cell 39: 925-938, 2010 .

25. Ma J, Wang P, Yao Y, Liu Y, Li Z, Liu X, Li Z, Zhao X, Xi Z, Teng $\mathrm{H}$, et al: Knockdown of long non-coding RNA MALAT1 increases the blood-tumor barrier permeability by up-regulating miR-140. Biochim Biophys Acta 1859: 324-338, 2016. 